\title{
Jumping Finite Automata for Tweet Comprehension
}

\author{
Stephen Obare \\ School of Computing \& IT \\ Jomo Kenyatta Universtiy \\ of Agriculture and Technology \\ Nairobi, Kenya. \\ smobareo@gmail.com
}

\author{
Abejide Ade-Ibijola \\ School of Consumer Intelligence \\ and Information Systems \\ University of Johannesburg \\ South Africa \\ abejideai@uj.ac.za
}

\author{
George Okeyo \\ School of Com Sci \& Informatics \\ De Montfort University \\ The Gateway, LE1 9BH \\ Leicester, United Kingdom. \\ george.okeyo@dmu.ac.uk
}

\begin{abstract}
Every day, over one billion social media text messages are generated worldwide, which provides abundant information that can lead to improvements in lives of people through evidence-based decision making. Twitter is rich in such data but there are a number of technical challenges in comprehending tweets including ambiguity of the language used in tweets which is exacerbated in under resourced languages. This paper presents an approach based on Jumping Finite Automata for automatic comprehension of tweets. We construct a WordNet for the language of Kenya (WoLK) based on analysis of tweet structure, formalize the space of tweet variation and abstract the space on a Finite Automata. In addition, we present a software tool called Automata-Aided Tweet Comprehension (ATC) tool that takes raw tweets as input, preprocesses, recognise the syntax and extracts semantic information to $86 \%$ success rate.
\end{abstract}

Keywords: Jumping finite automata, preprocessing, frontend compiler analysis, tweet comprehension

\section{Introduction}

Social media sites like Twitter have become increasingly popular in recent years with huge volumes of user-created content (UCC) in the form of text, social connection data, photos and videos ${ }^{1}$. Twitter, for instance, has over 974 million $^{2}$ subscribers and continues to grow both in size and activity [4]. Automatically comprehending this rich UCC can yield valuable information for a number of applications [1].

One such application is visualizing crime trends in a city to improve situational awareness [3], [2]. Property investors, for example, are keen to invest in areas that are relatively safe since tenants prefer safe neighbourhoods ${ }^{3}$. Twitter is

1. https://www.statista.com/topics/1164/social-networks/

2. https://www.cbsnews.com/news/many-twitter-users-dont-tweet-findsreport/

3. https://www.homes.com/blog/2016/05/secure-new-home-researchcrime-rates-impact-home-value/ rich in such information but is faced with a number of challenges which include ambiguity of the language in which tweets are written, high volumes, large number of spelling and grammatical errors, abbreviations, slang, meaningless information, the use of improper sentence structure [5], [7]. Manually sifting through these massive volumes of data to find information that is most useful is time consuming. Machine learning techniques have been used to compehend tweets with varied results [4]. Supervised learning techniques have primarily been applied in detecting small scale events e.g., civil unrests with the requirements of costly non-automatic data labeling which is labour intensive and time consuming [19]. Comprehension tasks that have no readily available labeled training data, are voluminous and noisy in nature like Twitter is the norm in todays computing environment and not the exception [6].

For detecting general and large scale events, unsupervised learning techniques which involves a set of documents with no further knowledge about the set has been used [27]. A model built upon such a set tries to find similarities and differences between the documents and separates them into clusters, where documents within each cluster are as similar as possible, and as different as possible between the clusters [26]. These clusters, however, do not have any real meaning. They are build on the observed features of the document set. One needs to interpret them to get useful results [24].

Beyond the scalability issues, the efficiency requirement is a pragmatic challenge for tweet processing. The processing speeds of existing NLP tools are often not up to the data generation speed e.g., 650 million Twitter messages per day $^{4}$. As a result, the efficiency gap between data generation and processing restricts the effectiveness of Twitter data processing for many real world applications. Automatically extracting actionable information from this type of data is an active research area in the domain of natural language processing (NLP) [25].

This paper presents a new approach based on formal language and automata theory to the tweet comprehension problem. The approach includes a Kenyan WordNet for

\footnotetext{
4. http://www.internetlivestats.com/twitter-statistics/
} 
improving the utility of tweets through ambiguity resolution, a formalized space of tweets variations and a JFA based tool that takes raw tweets, recognizes events of interest (syntax and semantics) and uses the derived insights to annotate maps. As a result this paper makes three key contributions:

1) WoLK - a novel lexical semantic knowledge base for Kenyan language that bridges the gap between WordNet ${ }^{5}$ and domain knowledge,

2) Formalized tweet space - generated a search space of possible variants of valid tweets that correctly identifies an event of interest, and

3) JFA - adapted methods from formal aspects of computing that are optimal for performing tweet comprehension task.

The rest of this paper is organised as follows. We present related work in Section 2. Section 3 specifies the design of WoLK and formalization of the space of tweet variation for abstraction onto a Finite Automata (FA). Section 4 demonstrates Automata-Aided ATC, a new tool that implements our technique while Section 5 presents conclusion and future work.

\section{Related Work}

This section presents a review of a number of language resources used in extracting semantics and techniques used in estimating similarities between sentences which are important for this research work.

\subsection{WordNets}

Over the past decades, the research community in the area of NLP have proposed a number of approaches for assigning senses to words based on the context of a sentence [32]. The approaches have lately been grouped into two main methodological approaches: knowledge-based and corpus-based algorithms [33]. Knowledge-based algorithms use lexical semantic resources to disambiguate words by defining explicit sense distinctions for assigning the correct sense of a word in context. Knowledge based algorithms give higher precision in disambiguating words in context but suffer from overlap sparsity and their performance depends largely on accuracy of dictionary definitions. Corpus-based methods use machine-learning algorithms which can either be supervised or unsupervised to disambiguate words from available sense inventory and annotated copra for the case of supervised learning and in the case of unsupervised learning where sense inventory and annotated copra is not required. Both knowledge-based and corpus-based algorithms present different benefits and drawbacks.

For knowledge based approaches, WordNet, which is a lexical semantic resource providing information about words with their meanings, has been widely used [4]. WordNet is currently the most advanced a lexical database created

5. https://wordnet.princeton.edu/ manually by English linguists providing an effective combination of traditional lexicographic information and modern computing.

In terms of structure, the main relation among words in WordNet is synonym. Nouns, verbs, adjectives, and adverbs are grouped into sets of cognitive synonyms called synsets which describes a distinct concept. Several other relations exist between synsets and words, such as antonymy hyponymy and meronymy as shown in Figure 1.

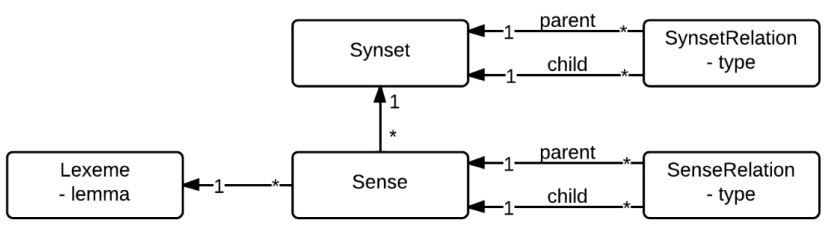

Figure 1: Structure of WordNet, Adapted from [9]

As observed by [10], manual construction of WordNet is a time consuming task and requires linguistic knowledge. In order to achieve comprehensive WordNet in languages other than English, two main approaches have been used [11]:

a). Merge approach - in this technique, an exhaustive repository of senses (meanings) of each word is compiled, synsets are then created that contain all of the applicable words for a given sense [12].

b). Expansion approach - existing synsets from a reference $\mathrm{WN}$ are used as a guide to create corresponding synsets in a new $\mathrm{WN}$, by gathering applicable words that represent the meaning of the synset. This approach has been shown to be suitable for under resourced languages [13].

Two methods have generally been used in expanding WordNets: automatically [17], and semi-automatically [18]. We highlight a number of WordNets developed based on expansion approach that are relevant to the development of a WordNet for the language of Kenya used in this work. The WordNets include: EuroWordNet developed by linking several European languages to English WordNet [14]; Persian WordNet [15]; Finnish WordNet [15]; Polish WordNet [16] and African WordNet (AWN) [13] created by aligning several languages spoken in Southern Africa. A number of tools were used in development of AWN including DEBVisDic ${ }^{6}$ editor tools for linguists building AWN.

\subsection{Estimating Sentence Similarity}

In how many ways can the same event be reported on Twitter? This is the question of variability [34]. Creativity is

6. https://deb.fi.muni.cz/proj_debvisdic.php 
highly involved in tweeting and two users are often likely to submit two different tweets [37]. If variability can be studied and modeled, then it becomes possible to build systems for comprehending tweets automatically, because such a system will be aware of every possible variation, and hence, will have the knowledge of the space of solutions.

[20] proposed a method to find similarities in sentences using Semantic Nets and Corpus Statistics. Their method was evaluated to be best suited sentences with short lengths. Sentence semantic similarity calculating method based on segmented semantic comparison was proposed in [30]. The achieved best results in short sentences. [28] described a metric method for computing sentence level semantic textual similarity based on a probabilistic finite state machine model that computes weighted edit distance. [8], [22] estimated context similarity based on closeness of semantic load of two comparing sentences. They built an FA through a systematic analysis of the patterns of meaning and use for each verb.

In this work we construct WoLK, a WordNet for the language of Kenya which consists of three parts: Princeton WordNet, local lexical dictionaries, (Kiswahili and Sheng) and annotated corpus in order to develop an initial lexicon and perform word-sense disambiguation. A linguistic expert reviewed the results to evaluate the method which gained a $70 \%$ accuracy. For estimating sentence similarities, we propose a method based on formalizing the space of tweet variation then abstracting and processing the tweet space through a FA.

\section{Semantics and Comprehension}

In this section, we describe the major components that constitute our technique.

\subsection{Definitions}

Definition 1 (Symbol, Alphabet, and String [21]). A symbol is a single token or word. An alphabet, denoted by $\Sigma$ is any finite set of symbols (words). A string is formulated from concatenation of zero or more symbols (words).

Definition 2 (Lexemes and Lexical Analysis [25]). Lexical analysis is the process of reading tweets and grouping them into "lexically meaningful" tokens referred to as lexemes.

\subsection{WoLK: A WordNet for the Language of Kenya}

We developed a framework for resolving ambiguity in tweets by first examining our Twitter corpora, paying particular attention to unknown tokens. Our experiment involved 28,361 which we manually annotated revealed that 12,613 tokens $(44.47 \%)$ were regarded as unknown by WordNet, out-of-vocabulary (OVV) and 14,168 tokens (49.96\%) were considered as in-vocabulary (IV) indicating the need to integrate more domain knowledge in WordNet knowledge base.

\subsection{Modelling Variability in Tweets}

A simple event of interest can be reported in quite a number of ways, each unique way being a mere variation resulting from valid permutation of tokens of interest. We formalised a novel approach to determine the variations (alternate and equivalent ) of any given tweet that helps us to automatically determine if a new tweet describes the same event.

$$
\begin{aligned}
& \text { Let }\left\{S_{1}, S_{2} \ldots S_{n}\right\} \text { be a set of tweets reporting } \\
& \text { the same event. } \\
& S_{1} \equiv S_{2} \equiv \ldots \equiv S_{n} \\
& \text { if and only if they are recognized by some } \\
& \text { automata. }
\end{aligned}
$$

$S_{1}$ is the base tweet (the primarily known event tweet from which other equivalent tweets are compared and/or derived). If $t w t_{(s 1)}$ is the base tweet and $\Sigma_{s}$ the alphabet of semantic tokens, then the language of all solutions to the tweet comprehension problem (which includes the base tweet), over $\Sigma_{s}$ is given as:

$$
\begin{aligned}
& L_{s}=\left\{t w t_{(s 1)}\right\} \cup\left\{L_{(t w t)}\right\} \\
& L_{s}=\left\{t w t_{(s 1)}\right\} \cup\left\{t w t_{1}, t w t_{2}, t w t_{3}, \ldots, t w t_{n}\right\}
\end{aligned}
$$

We present an algorithm based on the formalisms that creates a space of alternative solutions to the entire tweet comprehension problem by computing the concatenation of tokens in tweets. The space of solutions generated by the new algorithm represents the alternative ways a user may uniquely write a tweet describing the same event but making different choices of language constructs while exercising his/her limited or extensive knowledge of the rules of discourse. The idea is to take tweet seeds and then generate variations based on the seed from the product of its parts (semantic tokens).

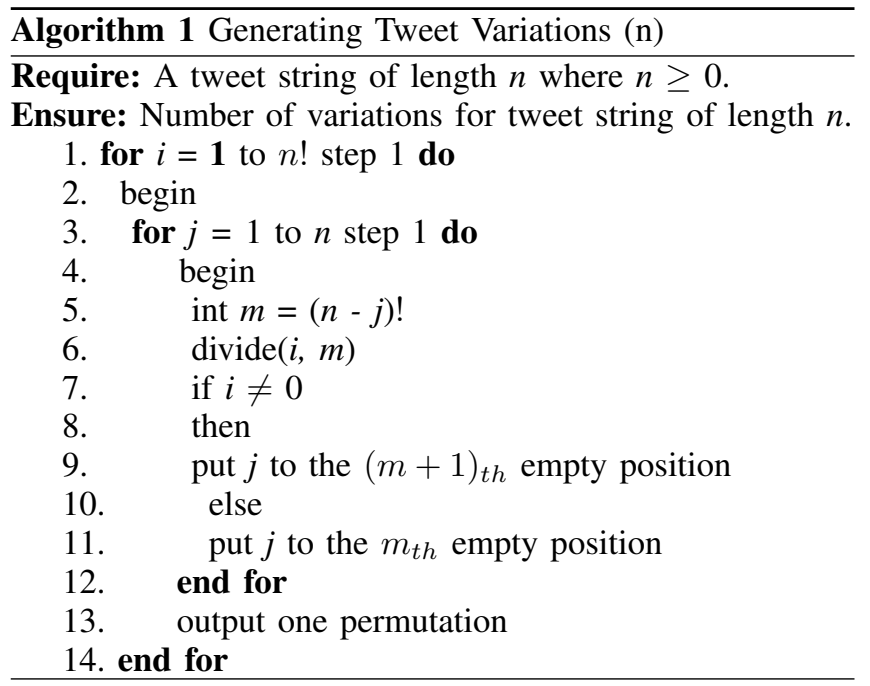




\subsection{Abstracting the Space of tweet Variation}

In [39], abstraction is described as the process of removing characteristics from a data set, in order to reduce it to a set of essential characteristics. We abstract the space of tweet variations onto an FA as a step towards comprehending tweets.

3.4.1. The States. We represented states by alphabets. Based on works by [31], events involve various participants and attributes and form a semantic (argument) structure: who did what to whom where and when. We represent a sample of these structure that forms our semantic tokens below. We identified these five entities that make up our alphabets in each tweet from the corpus.
who
what
whom
where
when

Given that:

$$
\begin{aligned}
& \sum_{\text {who }}={ }_{i} \\
& \sum_{\text {what }}={ }_{j} \\
& \sum_{\text {whom }}={ }_{k} \\
& \sum_{\text {where }}{ }^{=}{ }_{l} \\
& \sum_{m}
\end{aligned}
$$

Our state can therefore be treated as the combination of symbols/alphabets, whether single or multiple which is ${ }_{i},{ }_{j}$, $k, l, m=a_{0}, b_{1}, c_{2}, \ldots q_{i}$

Three gangsters were shot dead by policemen at Ongata Rongai

Two gangsters were shot dead near Langata by police men?

City MCA had dinner before killing his wife in a horror incident

Car jacking incident in Nyeri

Police kill a gangleader in Nairobi

A Woman has been sentenced to death for trafficking drugs in Gesonso Kisii county.

Youths are barricading the road at Southlands affecting motorists

3.4.2. Transition Process. Each tweet from Section 3.4.1 contains $\Sigma$. As depicted in Figure 2, we modelled our transition process using graph traversal.

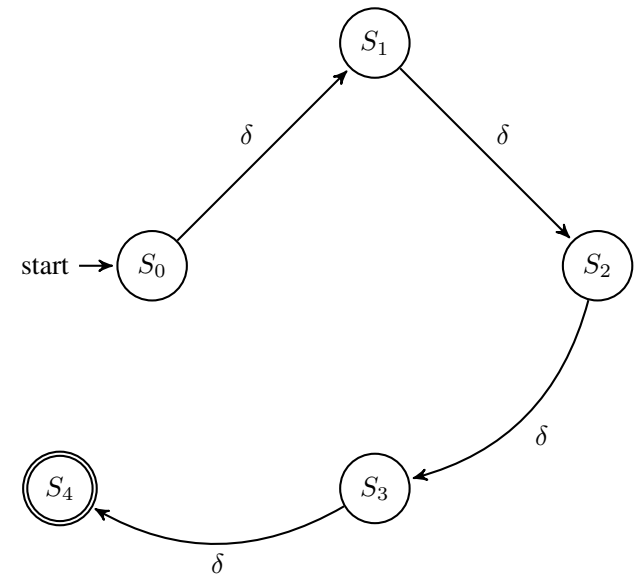

Figure 2: Transition process

\subsection{Comprehension using JFA}

We integrated the concepts described in Sections 3.2, 3.3 and 3.4 in our JFA technique. Within a particular running process in JFA, a computational step may be performed anywhere within a tweet string [21]. Therefore, before the next step is carried out, the process may jump over a large portion of the tweet string to the desired position of execution.

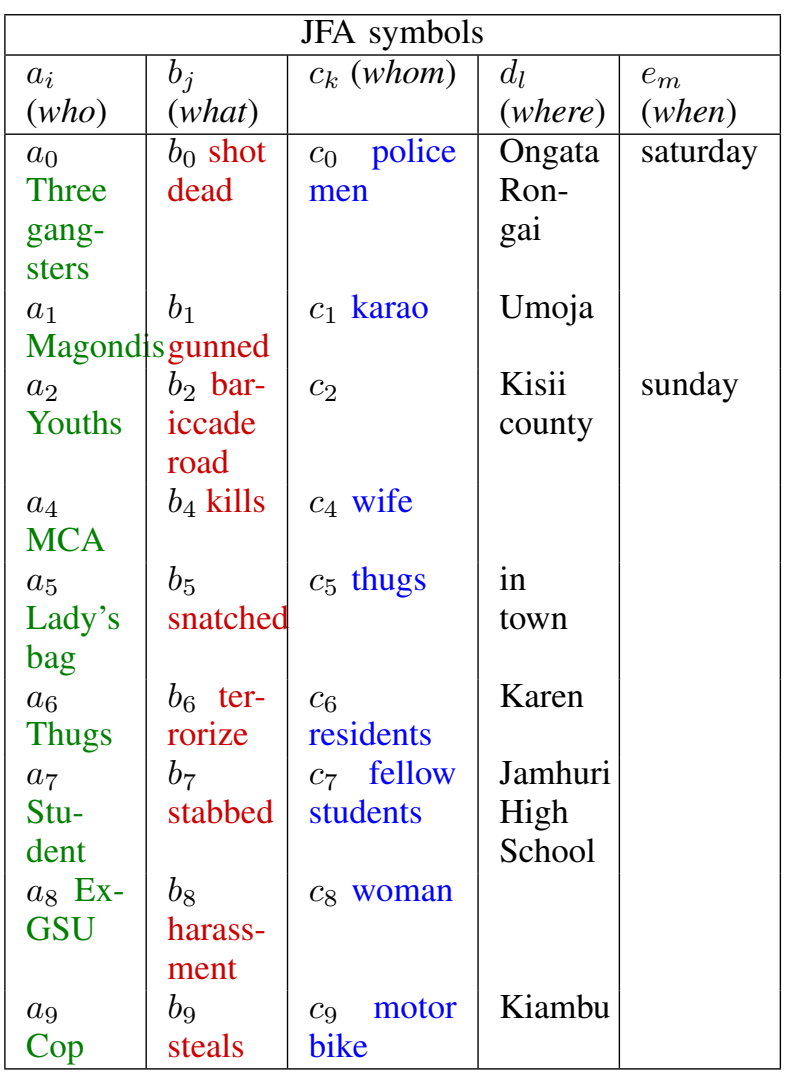

TABLE 1: JFA symbols table

The tweet of Example 1 contains five JFA symbols (semantic tokens) from the symbols table that are important 
for our comprehension task namely:

a. magondis, b. gunned, c. karao, d. Umoja.

These tokens can be matched to the set of alphabets

$$
\{a, b, c, d\}
$$

\section{Example 1.}

Three suspected magondis gunned in Umoja

Nairobi in shootout with Karao arrived...

AK-47 magazine, bundee, ignition switches

recovered

Following works by a number of researchers on the effect the following factors when reporting an event: [35] on effect of spelling, [36] on effect of input devices, and [38] on effect of community, the tweet of Example 1 can be re-written as shown in Example 2:

\section{Example 2.}

This is getting out of control. kupigwa ngeta na wagondi $_{a}$ in between the dark alleys in Umoja . $_{\text {. }}$ Three $\operatorname{shot}_{b}$ today by police $c$ after stealing car.

The needs to be cleared of thugs ${ }_{a}$ ASAP!......

We use (WoLK) developed in Section 3.2 as a comprehensive language resources for Kenyan language providing meaning to a number of tokens such as wagondi that helps us decipher that tweet 1 and 2 refer to the same event.

Given the sample tweet in Example 1 and based on our new formalization of variability described in Section 3.3, the space tweet variability can be given as

$$
\Sigma t w t_{i}, i>0=4 !=24 \text { possibilities. }
$$

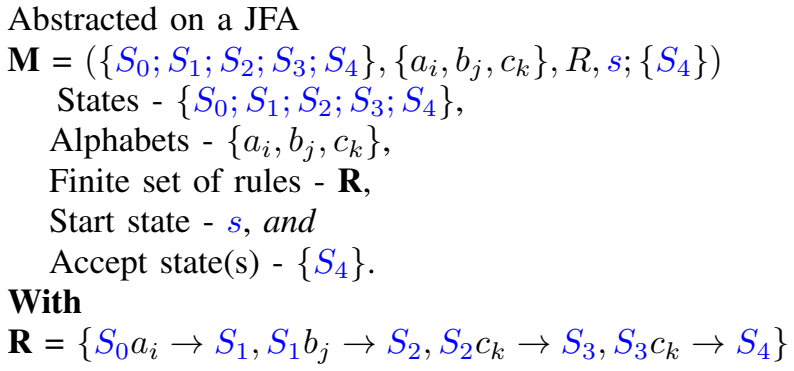

with the transition

$$
\begin{aligned}
b_{j} a_{i} c_{k} b_{j} c_{k} S_{0} a_{i} & \curvearrowright b_{j} a_{i} c_{k} S_{1} b_{j} c_{k} & & {\left[S_{0} a_{i} \rightarrow S_{1}\right] } \\
& \curvearrowright b_{j} a_{i} c_{k} S_{1} c_{k} & & {\left[S_{1} b_{j} \rightarrow S_{2}\right] } \\
& \curvearrowright b_{j} a_{i} S_{2} c_{k} & & {\left[S_{2} c_{k} \rightarrow S_{3}\right] } \\
& \curvearrowright b_{j} a_{i} S_{3} & & {\left[S_{3} c_{k} \rightarrow S_{4}\right] }
\end{aligned}
$$

$\mathrm{L}(\mathbf{M})$ recognises/accepts the tweet string of Example 1.

$\mathrm{L}(\mathbf{M})=\left\{w \in\left\{a_{i}, b_{j}, c_{k}\right\}^{*}:\left|a_{i}\right|=1 \leq\left|b_{j}\right| \leq 3=1 \leq\right.$ $\left.\left|c_{k}\right| \leq 3\right\}$

$a_{i} ; b_{j} ; c_{k}$ are defined in the JFA table of tokens extracted from our tweet corpus.

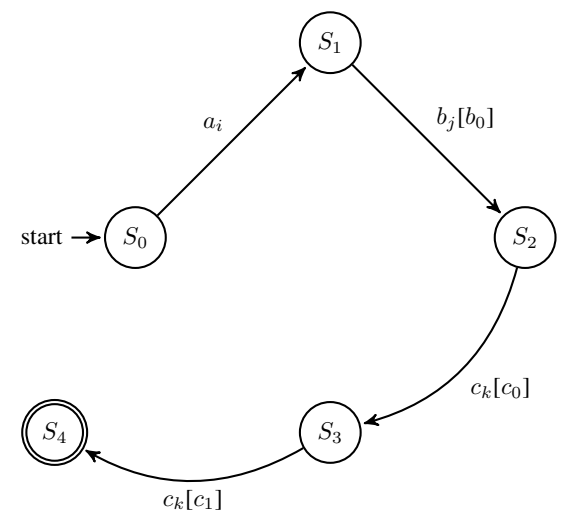

Figure 3: Transition Diagram tweet string in Example 1

\section{Implementation}

\subsection{ATC Tool}

We describe components of our approach which we developed for automating tweet comprehension. The essence of the system, as depicted in Figure 4, is an underlying preprocessing module which filters out noise before comprehension by a repository of JFAs.

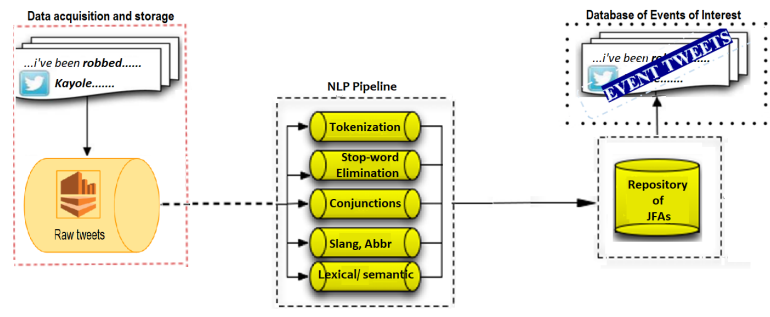

Figure 4: ATC System Architecture

We used Twitter streaming API to obtain tweets with geographic location and stored them in a data base of raw tweets. These raw tweets were then preprocessed using a number of techniques including the normalization algorithm 2 to make them emanable for our JFA technique.

Preprocessed tweets are then passed through a repository of JFAs for comprehension. Depending on the event of interest, the list of relevant tweets are then stored in a database of tweets of interest. The process is briefly illustrated in Algorithm 3.

\subsection{Testing}

We tested ATC with tweets retrieved from Nairobi city between September and December 2017. This history covers a period of four months and comprised 31,225 tweets from various crime hashtags. After preprocessing, (filtering retweets, stop word, abbreviations elimination, resolving slang using WoLK), the number of tweets reduced to 11,651 . 

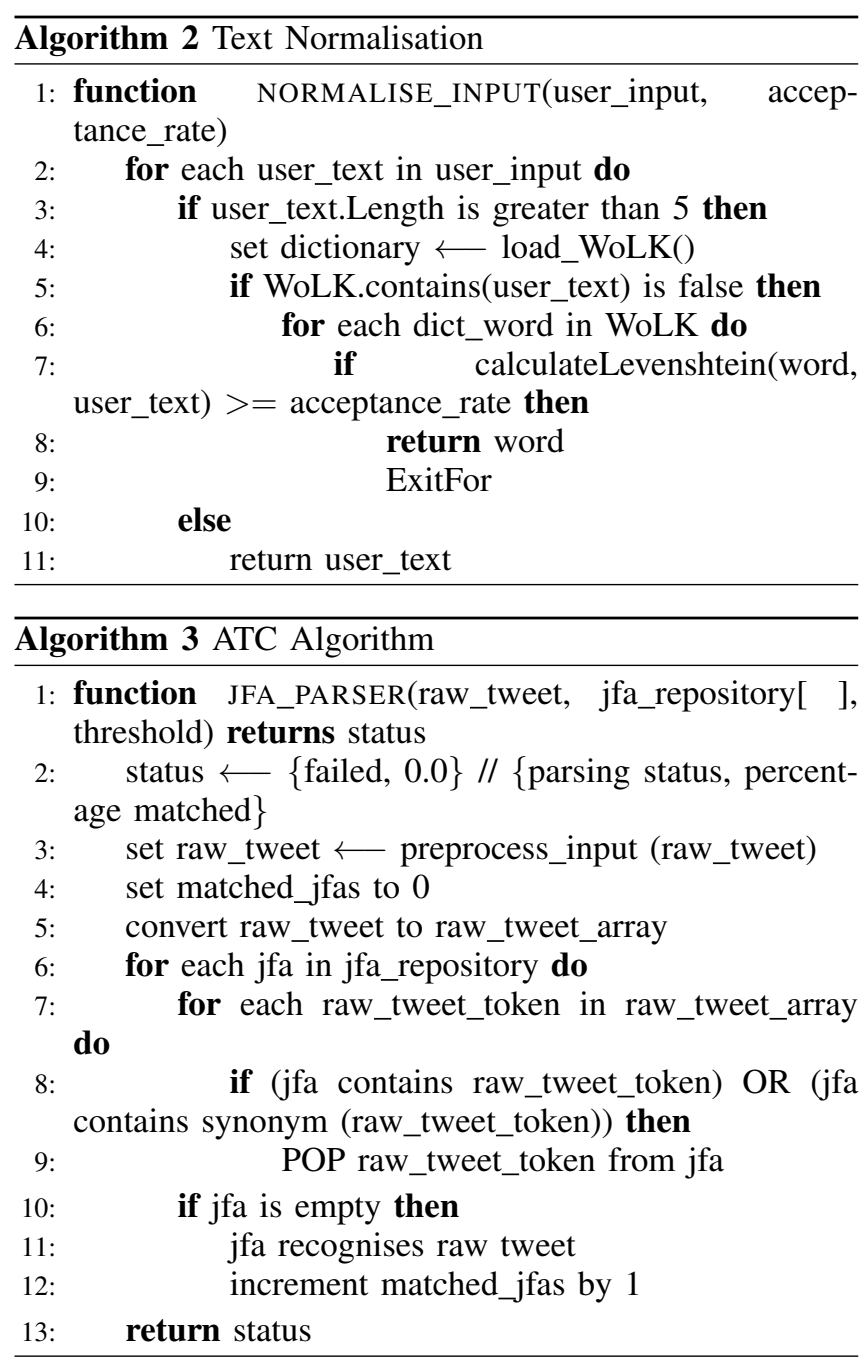

\subsection{Results}

We present the performance analysis of ATC, based on the 11,651 tweets from the perspective of accuracy in recognising tweets of interest. ATC failed to recognize only 1,631 hence the recognition accuracy of ATC is $86 \%$. Not all tweets that were not recognized are crime tweets and we attributed the failure to recognize some crime tweets to the non-exhaustive nature of WoLK knowledge base.

\section{Conclusions and Future Work}

The ATC tool provides automated tweet comprehension platform for tasks that require shifting through massive volumes of unstructure data. We started by developing a novel lexical semantic knowledge base to help us increases the coverage of WordNet and interpret tokens of interest, formalized the space of tweet variation and abstracted this space on a JFA as core components of ATC tool.

Tested on 31,225 tweets, ATC recognised $86 \%$ of our test data set. The $14 \%$ unrecognized tweets is significant and we will expand the coverage of ATC so that it can understand more of the event tweets.

\section{References}

[1] W. Maximilian, K. Michael: Geo-spatial event detection in the twitter stream, European conference on information retrieval, pages 356-367, 2013 , Springer.

[2] W. Willi, E. Jan-Philipp: Crime Mapping for Urban Planning-a Useful Tool for New Planning Times?, REAL Corp, 2013.

[3] W. Matthew L, B. Pete, S. Luke: Crime sensing with big data: The affordances and limitations of using open-source communications to estimate crime patterns, The British Journal of Criminology, volume 57:2, pages 320-340, 2017, Oxford University Press.

[4] A. Farzindar, K. Wael: A survey of techniques for event detection in twitter, Computational Intelligence, Volume 31:1, pages 132-164, 2015, publisher Wiley Online Library.

[5] D. Xiaowen, M. Dimitrios, C. Francesco, F. Pascal: Multiscale event detection in social media, Data Mining and Knowledge Discovery, volume 29: 5, pages 1374-1405, 2015, Springer.

[6] M. Banko, Eric. B: Scaling to very very large corpora for natural language disambiguation, Proceedings of the 39th annual meeting on association for computational linguistics, pages 26-33, 2001, Association for Computational Linguistics

[7] S. Hassan, H. Yulan, A. Harith: Alleviating data sparsity for twitter sentiment analysis, 2012, CEUR Workshop Proceedings (CEUR-WS. org).

[8] Mohammadi, Mehdi and Fakhrahmad, SM: Effective estimation of context similarity: A proposed matching model based on weighted semantic load, journal=International Journal of Artificial Intelligence \& Applications, volume $=3$, number $=3$, pages $=1$, year $=2012$, publisher=Academy \& Industry Research Collaboration Center (AIRCC)

[9] Redkar, Hanumant Harichandra and Bhingardive, Sudha Baban and Kanojia, Diptesh and Bhattacharyya, Pushpak: World WordNet database structure: an efficient schema for storing information of WordNets of the world, Twenty-Ninth AAAI Conference on Artificial Intelligence, 2015.

[10] Miller, George: WordNet: An electronic lexical database, year=1998, publisher=MIT press

[11] Vossen, PJTM, EuroWordNet: general document, year 2002, publisher Amsterdam: Vrije Universiteit.

[12] Griesel, M. and Bosch, S., Taking stock of the African Wordnet project: 5 years of development, in $\mathrm{H}$. Orav, C. Fellbaum and $\mathrm{P}$. Vossen (eds.), Proceedings of the 7th Global WordNet Conference, 2014 (GWC2014), Demonstration Session, Tartu, Estonia, January 2529, pp. 148-153.

[13] Bosch, Sonja E and Griesel, Marissa Strategies for building WordNets for under-resourced languages: The case of African languages, journal of Literator, volume $=38$, number $=1$, pages $=1-12,2017$, AOSIS Publishing.

[14] Huang, Chu-Ren and Calzolari, Nicoletta and Gangemi, Aldo, Ontology and the lexicon: A natural language processing perspective, 2010 , Cambridge University Press.

[15] Griesel, Marissa and Bosch, Sonja, Taking stock of the African Wordnet project: 5 years of development, Proceedings of the Seventh Global Wordnet Conference, pages=148-153, 2014.

[16] Rudnicka, Ewa and Maziarz, Marek and Piasecki, Maciej and Szpakowicz, Stan, A strategy of mapping Polish Wordnet onto Princeton Wordnet, journal=Proceedings of COLING 2012: Posters, pages=1039-1048, 2012.

[17] Oliver, A., WN-Toolkit: Automatic generation of WordNets following the expand model, Proceedings of the 7th Global WordNet Conference 2014, Demonstration Session, Tartu, Estonia, January 25-29, pp. 7-15. 
[18] Hiroyuki Kaji and Mariko Watanabe. Automatic construction of Japanese WordNet. 6th International Conference on Language Resources and Evaluation (LREC), Genoa, Italy, May, 2006.

[19] S. Takeshi, O. Makoto, M. Yutaka: Earthquake shakes Twitter users: real-time event detection by social sensors, Proceedings of the 19th international conference on World wide web, pages 851-860, 2010, ACM.

[20] Li, Yuhua and McLean, David and Bandar, Zuhair A and O'shea, James D and Crockett, Keeley: Sentence similarity based on semantic nets and corpus statistics, journal=IEEE transactions on knowledge and data engineering, volume $=18$, number $=8$, pages $=1138-1150$, year=2006, publisher=IEEE

[21] M. Alexander, Z. Petr: Jumping finite automata, Regulated Grammars and Automata, pages 567-585, 2014, Springer.

[22] Popescu, Octavian: Learning corpus patterns using finite state automata, booktitle $=$ Proceedings of the 10th International Conference on Computational Semantics (IWCS 2013)-Long Papers, pages=191-203, year $=2013$

[23] Dao, Thanh Ngoc and Simpson, Troy: Measuring similarity between sentences, journal=The Code Project, year $=2005$

[24] P. Berkhin A survey of clustering data mining techniques, Grouping multidimensional data, pages 25-71, 2006, Springer

[25] A. Ade-Ibijola: FINCHAN: A Grammar-based Tool for Automatic Comprehension of Financial Instant Messages, Proceedings of the Annual Conference of the South African Institute of Computer Scientists and Information Technologists, pages 1, 2016, ACM.

[26] Cohn, David and Caruana, Rich and McCallum, Andrew: Semisupervised clustering with user feedback, Journal of Constrained Clustering: Advances in Algorithms, Theory, and Applications, volume $=4$, pages $17-32,2003$

[27] A. Foteini, M. Sebastian, R. Krithi, W. Gerhard: See what's enBlogue: real-time emergent topic identification in social media, Proceedings of the 15th International Conference on Extending Database Technology, pages 336-347, 2012, ACM

[28] Wang, Mengqiu and Cer, Daniel: Stanford: probabilistic edit distance metrics for sts, Proceedings of the First Joint Conference on Lexical and Computational Semantics-Volume 1: Proceedings of the main conference and the shared task, and Volume 2: Proceedings of the Sixth International Workshop on Semantic Evaluation, pages=648-654, year=2012, organization=Association for Computational Linguistics

[29] Carpenter, Patricia A and Miyake, Akira and Just, Marcel Adam: Language comprehension: Sentence and discourse processing, journal $=$ Annual review of psychology, volume $=46$, number $=1$, pages $=91-$ 120, year=1995, publisher=Annual Reviews 4139 El Camino Way, PO Box 10139, Palo Alto, CA 94303-0139, USA

[30] Liu, YUNTONG and Liang, Yanjun: A sentence semantic similarity calculating method based on segmented semantic comparison, journal=Journal of Theoretical and Applied Information Technology, volume $=48$, number $=1$, pages $=231-235$, year $=2013$, publisher $=$ Citeseer

[31] Gehrke, Berit: Adverbial functions of Slavic prefixes, booktitle $=$ Workshop on event structure in linguistic form and interpretation, Leipzig, year $=2004$, organization $=$ Citeseer

[32] Torres, Fredy: Designing and Developing a WSD model for NLP, pages $=71,2017$

[33] Mihalcea, Rada and Corley, Courtney and Strapparava, Carlo and others: Corpus-based and knowledge-based measures of text semantic similarity, $A A A I$, pages $=775-780,2006$

[34] Dagan, Ido and Glickman, Oren: Probabilistic textual entailment: Generic applied modeling of language variability, year=2004

[35] Chowdhury, Gobinda G: Natural language processing, journal=Annual review of information science and technology, volume $=37$, number $=1$, pages $=51-89$, year $=2003$, publisher $=$ Wiley Online Library
[36] Murthy, Dhiraj and Bowman, Sawyer and Gross, Alexander J and McGarry, Marisa: Do we tweet differently from our mobile devices? a study of language differences on mobile and web-based twitter platforms, journal $=$ Journal of Communication, volume $=65$, number $=5$ pages $=816-837$, year $=2015$, publisher $=$ Oxford University Press

[37] Rist, Robert S: Variability in program design: the interaction of process with knowledge, journal=International Journal of Man-Machine Studies, volume $=33$, number $=3$, pages $=305-322$, year $=1990$, publisher=Elsevier

[38] Eisenstein, Jacob and O'Connor, Brendan and Smith, Noah A and Xing, Eric P: A latent variable model for geographic lexical variation, booktitle=Proceedings of the 2010 Conference on Empirical Methods in Natural Language Processing, pages $=1277-1287$, year=2010, organization=Association for Computational Linguistics

[39] Kramer, Jeff: Is abstraction the key to computing?, author=Kramer, Jeff, journal=Communications of the $A C M$, volume $=50$, number $=4$, pages $=36-42$, year $=2007$, publisher $=\mathrm{ACM}$ 